\title{
Pruebas experimentales acerca de la capacitancia en el diseño y construcción de un condensador didáctico
}

\author{
Experimental trials about the capacitance in designing and \\ constructing of an educational capacitor
}

\author{
José Jadir Layton Corzo'
}

\section{Resumen}

Por medio del diseño y la construcción de un artefacto experimental y el uso del circuito electrónico Arduino como instrumento de medición, se realizó una serie de experimentos sobre la capacitancia de dos placas de cobre paralelas. Para los experimentos se desarrolló un condensador de placas paralelas móvil que funciona como una plataforma experimental, que sirve para realizar mediciones de la capacitancia variando su forma estructural en el área de sus placas, la distancia y el dieléctrico que las separa. Los valores de las mediciones en Pico, Nano y Microfaradios se pueden ver en una pantalla de un ordenador donde esté instalado el software Arduino, estos resultados experimentales se compararon con otros resultados teóricos previamente estudiados, y así llevar a cabo la práctica de laboratorio. Estos experimentos acercan al estudiante a una mayor comprensión de la materia y también sirve para profundizar en la investigación de aplicaciones instrumentales. La construcción de este prototipo lleva al estudiante del aprendizaje teórico de la materia a la experimentación y la búsqueda de aplicaciones de sus conocimientos, interactuando de manera didáctica con las variables presentes en este dispositivo.

\section{Palabras clave: campos electromagnéticos, condensadores, energía eléctrica, dieléctricas, potencial eléctrico (tesauro spines).}

\section{Abstract}

Through the design and construction of an experimental artifact and the use of the Arduino electronic circuit as a measuring instrument, a series of experiments were carried out on the capacitance of two parallel copper plates. For the experiments a mobile parallel plate capacitor was developed that works as an experimental platform, which serves to make measurements of the capacitance varying its structural form in its plate's area, the distance and the dielectric that divides them. The values of the measurements in Pico, Nano and Micro farads can be seen on a computer screen where the Arduino software has been installed previously, these experimental results were compared with other theoretical results previously studied and thus carrying out in laboratory practicals. These experiments allow students to get closer to a greater understanding of the subject as well as in deep research on instrumental applications. The construction of this prototype takes students from the theoretical learning of the subject to the experimentation and in the search of applications of their knowledge interacting in an educational way with the variables described in this device.

\section{Keywords: Electromagnetic fields, Capacitors, Electric power, Dielectric, Electric potential}

1 Estudiante Técnica Profesional en Electrónica Industrial Escuela Tecnológica Instituto Técnico Central. Integrante semillero de investigación en diseños experimentales DIEXP, jjlaytonc@itc.edu.co . 


\section{Introducción}

Por medio del diseño y la construcción de un artefacto experimental y el uso del circuito electrónico Arduino como instrumento de medición, se realizaron una serie de experimentos sobre la capacitancia de dos placas de cobre paralelas. El objetivo general es estudiar los fenómenos eléctricos por medio de plataformas didácticas para implementarlas en los laboratorios y en las clases de física eléctrica, esto se realizó en este caso para brindar una herramienta experimental para el estudio de la capacitancia de un condensador didáctico que también sirve para la enseñanza.

Los condensadores son dispositivos eléctricos comúnmente utilizados como componentes electrónicos implementados en circuitos osciladores, como por ejemplo, en sensores industriales para medir la presión, detección de objetos y proximidad, almacenamiento de energía eléctrica aplicada en los desfibriladores para resucitar pacientes, para sintonizar frecuencias en los radios, como filtros de voltaje y corriente al utilizarlos junto con un diodo, estas son algunas de sus muchas aplicaciones.

Este dispositivo generalmente está formado por dos placas de metal en paralelo separadas entre sí por un material dieléctrico a una distancia entre placas, las cuales están conectadas a dos terminales de una fuente eléctrica, cada una de carga positiva o negativa respectivamente, al aplicar un voltaje las cargas positivas y negativas que proporciona la fuente se atraerán entre sí, concentrándose en las superficies de las placas paralelas del condensador, debido a la fuerza eléctrica que experimentan las cargas de signo contrario, si por ejemplo acercamos un objeto eléctricamente cargado entre las placas del condensador sería atraído por cargas de signo opuesto que se encuentre en las placas, esto se debe a que la presencia de cargas acumuladas en el medio generan un campo electromagnético que atrae o repele objetos cargados, y se intensifica en cuanto mayor sea la carga acumulada.
La capacitancia en un condensador también aumenta si entre sus placas se dispone de un material dieléctrico para almacenar mayor campo eléctrico por medio de esta propiedad de los materiales Ilamada permitividad eléctrica relativa, y cada uno de los materiales dieléctricos tienen su propio valor, algunos ejemplos de estos materiales son: el aire, la mica y el papel (Sears, 2009).

La capacitancia eléctrica es la medida eléctrica de la capacidad de almacenamientos de un condensador, y se define también como la relación que hay entre el voltaje (V) aplicado en los bornes de la placa del condensador y la cantidad de carga (q) almacenada en sus placas, esta carga se encuentra relacionada con la cantidad de campo eléctrico, entonces la capacitancia es directamente proporcional al área de las placas de los condensadores, pero disminuirá a medida que la distancia entre las placas aumente, el condensador también tiene un tiempo de carga y descarga comúnmente llamado Tau $(\tau)$, que generalmente está relacionado en una ecuación de respuesta natural de una capacitancia (Rela, 2010). Los valores de las mediciones en pico, nano y microfaradios se pueden ver en una pantalla de un ordenador donde esté instalado el software Arduino, estos resultados experimentales se comparan con otros resultados teóricos previamente estudiados, y así llevar a cabo la práctica de laboratorio.

\section{Metodología}

Los materiales usados para el prototipo fueron: placas de cobre o baquelitas, tablas de madera, corredera, motor, placa electrónica Arduino, base plástica.

\section{Procedimiento}

Inicialmente la variable a controlar en el condensador didáctico fue la distancia entre las placas, porque era lo más conveniente para realizar experimentos con péndulos de aluminio cargados 
eléctricamente, y a su vez utilizarlo para medir la capacitancia entre las placas, para ello se diseñó un soporte con una base móvil, que permite acercar o alejar las placas entre sí, como se muestra en la figura 1, las bases que sostienen las placas cuentan con un espacio que puede ser reducido por medio de dos tornillos y así sostener las placas, esto permite intercambiarlas para experimentar con diferentes tipos.

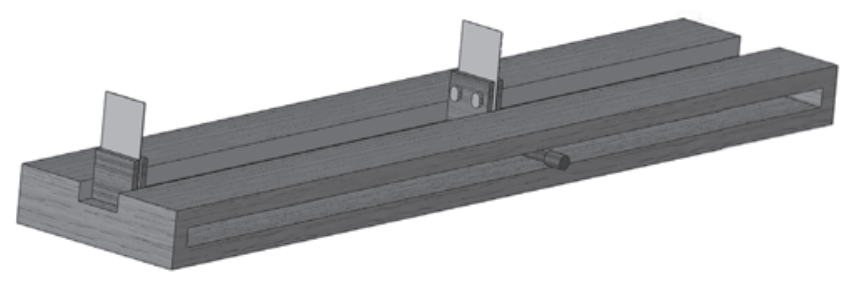

Figura 1. Primer diseño de base experimental.

Fuente. autor

Para medir el mayor rango de capacitancia posible en el condensador, se presentó un problema con los tornillos, debido a que estos ocupan un espacio considerable para la medición, y se requirió que la distancia entre placas sea la menor posible, para esto se desarrolló otro diseño que soluciona el anterior problema, utilizando en su lugar un par de paletas como soporte para las placas y cinta doble faz como adhesivo que las sostiene, esta solución cambia el diseño agregando tan solo un material adicional que permite realizar los cambios de placas para diferentes experimentos. Ver figura 2.

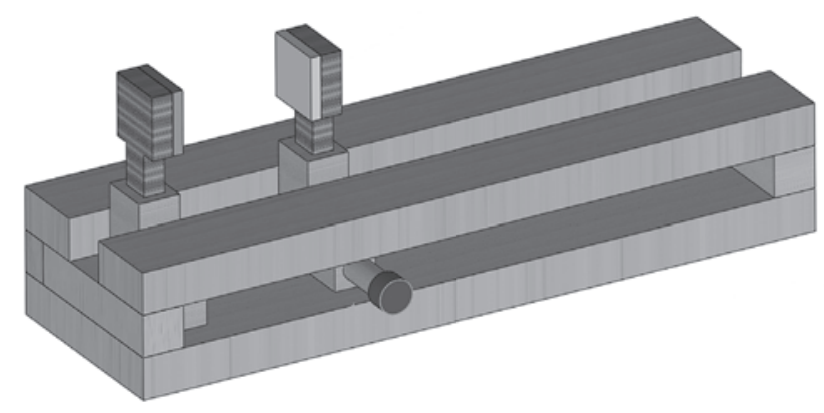

Figura 2. Segundo diseño de base experimental. Fuente Autor
Para medir y visualizar la capacitancia del condensador didáctico se investigó sobre el diseño de un circuito medidor de capacitancia con Arduino, una plataforma de hardware libre basada en una placa con un microcontrolador y un entorno de desarrollo también utilizada con fines didácticos. En internet se pueden encontrar varios diseños de medidores de capacitancia, en este caso se utilizó el amplificador operacional TL081 para el circuito de medición que se muestra en la figura 3.

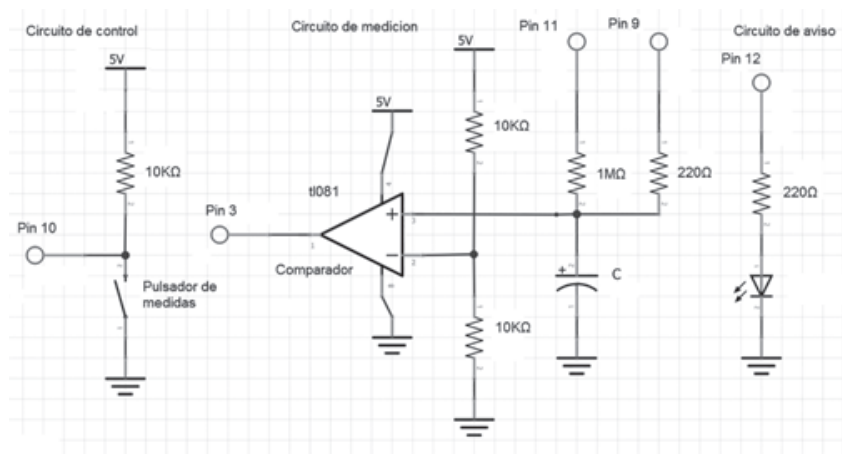

Figura 3. Circuito medidor de capacitancia.

Fuente el autor

El circuito medidor cumple la función de comparar las dos entradas de voltaje del amplificador, la entrada inversora representada con signo (-) está conectada a un divisor de voltaje que es utilizado como referencia y tiene un valor de $2.5 \mathrm{~V}$, en la entrada no inversora con signo (+) está conectado un circuito RC que será el objeto principal de nuestra medición y donde se conecta el condensador didáctico (C). El circuito también cuenta con un pulsador que inicia la medición y un LED que avisa cuando el condensador está cargando, todo el circuito puede ser alimentado con Arduino, que además lleva cuenta del periodo de carga (t) y el cálculo de la capacitancia por medio de la ecuación de valores iniciales que se muestran en la figura 4, donde la capacitancia es la variable dependiente y el tiempo de carga la variable independiente en la medición.

La programación del microcontrolador Arduino como medidor de capacitancias se puede dividir 
en un algoritmo de tres partes, donde inicialmente se declaran las variables que se van a utilizar para el cálculo del tiempo de carga del condensador, el programa espera la señal del interruptor en un bloque condicional para iniciarlo, si el interruptor es presionado el condensador es totalmente descargado por medio del pin 9 y la resistencia de $220 \Omega$, para que después de un tiempo de espera el condensador comience a cargarse por medio del pin 11 y tome registro del tiempo de inicio de carga, como se muestra en la figura 4. Luego el programa espera el momento en el que el voltaje del condensador supere el voltaje de referencia, que es seguido por un aumento del voltaje de la salida del amplificador operacional conectado al pin 3 del Arduino, cuando esto ocurre el microcontrolador detiene las funciones y registra el tiempo de carga del condensador, para luego pasarlo por un filtro de bloques condicionales del programa que permite obtener una medición más confiable.

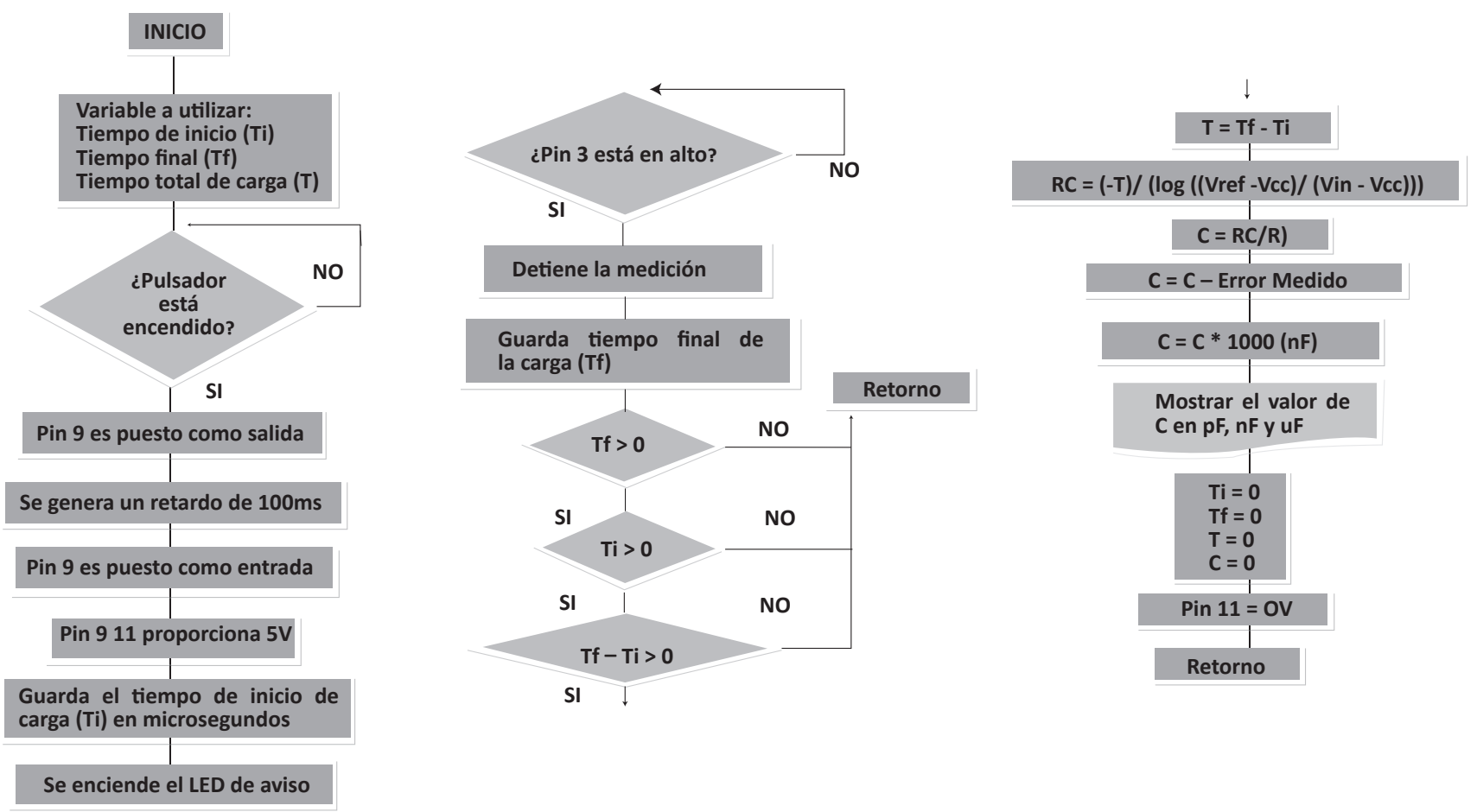

Figura 4. Diagramas de flujo del programa medidor de capacitancia.

Por último, el microcontrolador calcula la capacitancia con el tiempo de carga del condensador y restándole un valor capacitivo de error, el cual se halla tomando medidas sin ningún capacitor y en este caso tiene un valor de 52.5 picofaradios, el valor obtenido se muestra en la pantalla del computador en el orden de picofaradios, nano faradios, y microfaradios, después se borran los valores de las variables para iniciar con otra medición (figura 8). Antes de iniciar con los experimentos se hicieron mediciones con condensadores del mercado para corroborar el buen funcionamiento de medidor de capacitancia, el cual resultó muy útil para llevar a cabo laboratorios con circuitos de condensadores en serie y paralelo. Se experimentó inicialmente con dos placas de cobre utilizando el aire como dieléctrico y la distancia que separa las placas como variable independiente, 
se calculó el valor de una constante con los valores conocidos de la ecuación de capacitancia en relación con la estructura del condensador sin variar en este caso el tamaño de las placas, los resultados se muestran en la Tabla 1 . En el segundo experimento se utilizaron hojas de papel cuadriculadas de un block como dieléctrico entre las placas del condensador didáctico, se calculó el grosor de las hojas doblándose hasta medir un milímetro y dividiendo después por el número de hojas. Se obtuvo una ecuación de capacitancia similar a la anterior, pero en este caso el valor de la permitividad eléctrica del papel es mayor y la distancia como factor del número de hojas que se utilizaron por su espesor obteniendo como resultado los valores de la tabla 2.

Tabla 1. Datos experimentales con dieléctrico de aire. Fuente los autores

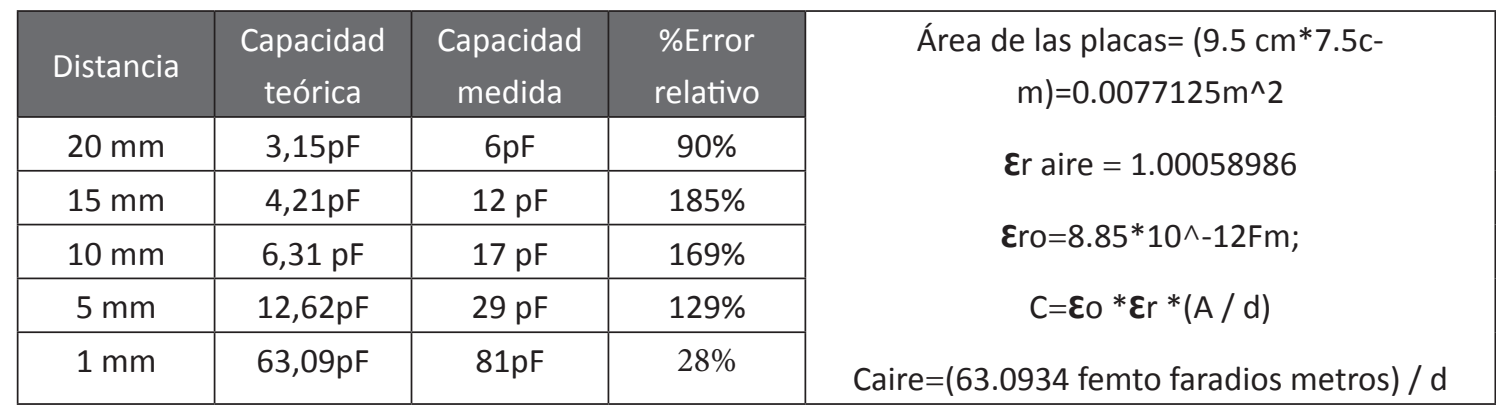

Tabla 2. Datos experimentales con dieléctrico de papel. Fuente los autores

\begin{tabular}{|c|c|c|c|c|}
\hline $\begin{array}{l}\text { No. } \\
\text { hojas }\end{array}$ & $\begin{array}{l}\text { Capacidad } \\
\text { Teórica }\end{array}$ & $\begin{array}{l}\text { Capacidad } \\
\text { medida }\end{array}$ & $\begin{array}{l}\text { \% Error rela- } \\
\quad \text { tivo }\end{array}$ & $\begin{array}{l}\text { Área de las placas }=(9.5 \mathrm{~cm} * 7.5 \mathrm{c}- \\
\qquad \mathrm{m})=0.0077125 \mathrm{~m}^{\wedge} 2\end{array}$ \\
\hline 1 & $756 \mathrm{pF}$ & $854 \mathrm{pF}$ & $13 \%$ & $\varepsilon r o=8.85^{*} 10^{\wedge}-12 \mathrm{Fm}$ \\
\hline 2 & $756 \mathrm{pF}$ & $629 \mathrm{pF}$ & $66 \%$ & Er papel = 1.5 \\
\hline 3 & $252 \mathrm{pF}$ & $439 \mathrm{pF}$ & $74 \%$ & $C=\varepsilon_{0} * \varepsilon r *(A / d)$ \\
\hline 4 & $189 \mathrm{pF}$ & $335 \mathrm{pF}$ & $77 \%$ & Cnond - 101581275 fomto faradios motrol / \\
\hline 5 & $151 \mathrm{pF}$ & $306 \mathrm{pF}$ & $103 \%$ & Cpapel $=(94.5843 / 5$ femto faradios metros $) / \mathrm{d}$ \\
\hline 6 & $126 \mathrm{pF}$ & $271 \mathrm{pF}$ & $115 \mathrm{pF}$ & Grosor papel $=125$ micra metros \\
\hline
\end{tabular}

Equipo: computadora con el software Arduino.

\section{Resultados}

Como resultado final se construyó el prototipo que aparece en la figura 5 , se comprobó la versatilidad adhesiva de la cinta para cambio y soporte de las placas, también se utilizó en otros experimentos con péndulos de aluminio y las placas conectadas al generador de Wimshurst para observar los efectos del campo eléctrico, este experimento también se llama las campanas de Franklin.
Por medio de este dispositivo se pueden llevar a cabo prácticas de laboratorio donde los estudiantes podrán ver los efectos del campo eléctrico presentes en las fuerzas y energía eléctrica generada entre placas. Es por esta razón que se realizó la labor de diseñar y construir un dispositivo que sirve para la recreación del funcionamiento de un condensador con objetivos didáctico en la materia de física eléctrica, y en donde intervienen temáticas de conocimientos en electrónica, programación y un poco de diseño de mecanismos. Figuras 5 y 6 . 


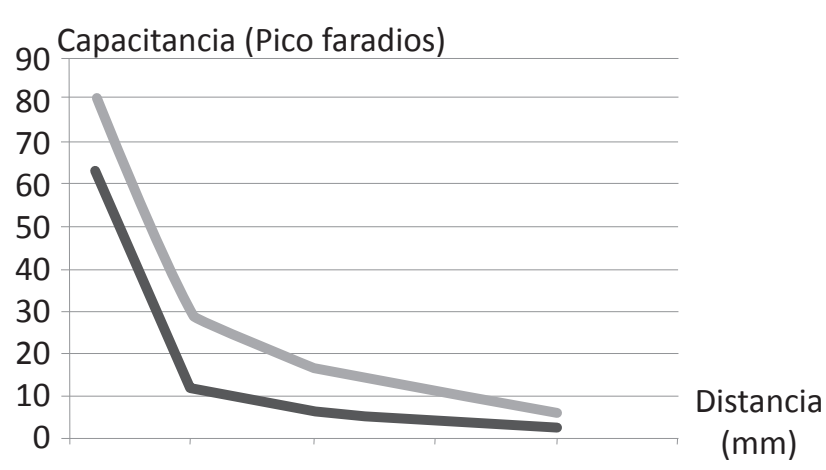

Figura 5. Experimento con aire. Fuente autores

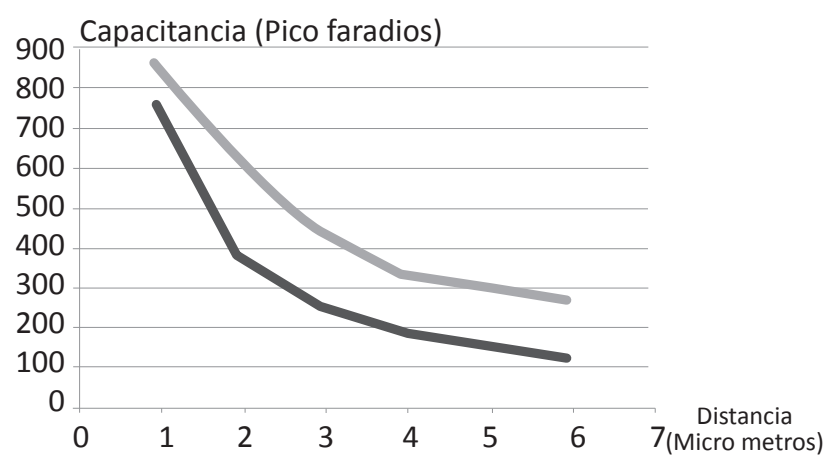

Figura 6. Experimento con papel. Fuente autores

Con este dispositivo experimental y la utilización de la plataforma Arduino, el estudiante puede experimentar con el condensador didáctico visualizando en un computador la medida en unidades de faradios y la capacitancia entre sus placas, realizada por un circuito amplificador operacional y una placa con microcontrolador Arduino que sirve como medidor de capacitancia, el estudiante puede utilizar como variables independientes en sus experimentos la distancia entre placas, material, dieléctrico y área. Figura 7.

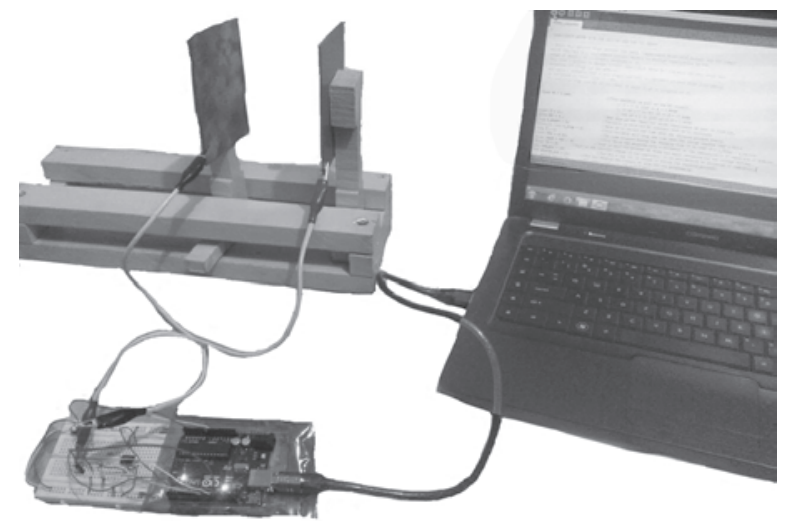

Figura 7. Prototipo experimental inicial. Fuente autores

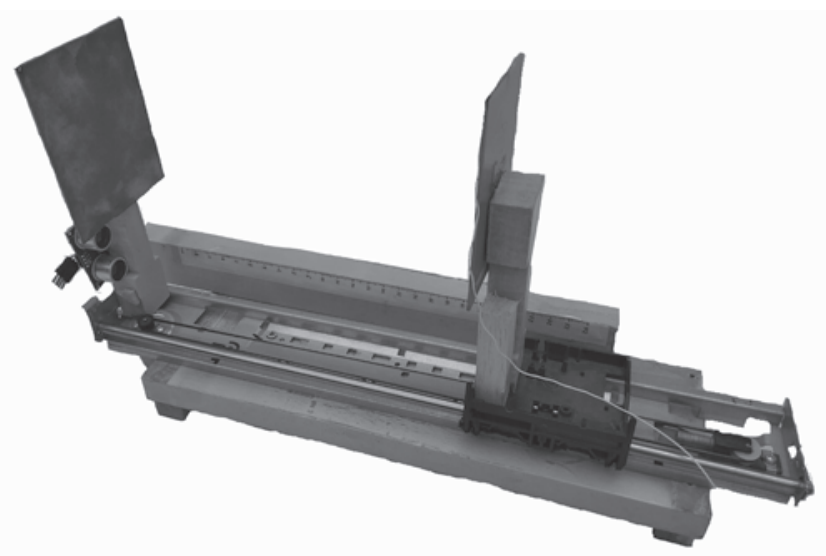

Figura 8. Prototipo experimental con sensores y motor. Fuente autores

Estos conceptos físicos están respaldados por modelos matemáticos vistos en el curso de física eléctrica que pueden predecir el comportamiento de los dispositivos eléctricos y electrónicos, y dependen principalmente de variables independientes estrechamente relacionadas con el comportamiento eléctrico del dispositivo, como por ejemplo en el caso del condensador didáctico propuesto por el semillero, sus variables independientes son la distancia entre las placas paralelas, material y áreas de las placas, la variable dependiente en este caso es la capacitancia del condensador. Esto permite la posibilidad de llevar a cabo más que una simple práctica, la experimentación de los estudiantes utilizando distintos materiales con el uso de los conocimientos en la electrónica y la programación para la visualización de datos de las variables dependientes o a medir, y para la corroboración del modelo matemático con el fenómeno real. "Sin embargo, muchos estudiantes piensan que el propósito del trabajo de laboratorio es seguir instrucciones y obtener la respuesta correcta, por lo que se concentran en la idea de manipular instrumentos más que manejar ideas" (Cardona, 2013, p.)

\section{Conclusiones}

Se construyó un prototipo de un condensador didáctico con un medidor de capacitancia muy 
útil para la experimentación de la capacitancia, se experimentó en el prototipo con dos modelos matemáticos de la capacitancia de un condensador con relación al tiempo de carga y la formación estructural de sus placas, dando como resultado una aproximación en las medidas, aunque no son precisas, son muy aproximadas a los valores y patrones esperados en el comportamiento de este fenómeno eléctrico. Cabe resaltar que el prototipo puede ser utilizado para el estudio en la materia de física eléctrica, como también para el repaso de conceptos de programación con aplicación en instrumentación y la aplicación de los amplificadores operacionales, junto con los circuitos RC en electrónica.

\section{Referencias bibliográficas}

Cardona, F (2013). Las prácticas de laboratorio como estrategia Didáctica. Santiago de Cali, Colombia: Instituto de Educación y Pedagogía.

Rela, A. (2010). Electricidad y electrónica. Buenos aires, Argentina: Instituto Nacional de Educación Tecnológica.

Sears, Z. (2009). Física universitaria con física moderna. México. Pearson. 